\title{
. MASTER
}

FUNCTIONAL INTEGRAL APPROACH TO "CLASSICAL STATISTICAL DYNAMICS

BY

R. V. JENSEN

\section{PLASMA PHYSICS LABORATORY}

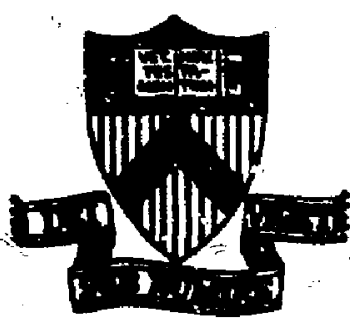

\section{PRINCETON UNIVERSITY PRINCETON, NEW JERSEY}

\footnotetext{
This work we support od by" the U.S. Depertient of Energy Contract Ho. OE-ACO2-76-CHO 3073. Auproduce ion, trans Iaiton, publicition, use and disposelp in whols or in purt,

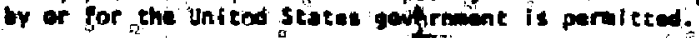


FUNCTIONAL INTEGRAL APPROACH TO CLASSICAL STATISTICAL DYNAMICS

Roderick V. Jensen

Plasma Physies Laboratory, Princeton University, Princeton, New Jersey 08544

A functional integral method is developed for the statistical solution of nonlinear stochastic differential equations which arise in classical dynamics. The functional integral approach provides a very naturai and elegant derivation of the statistical dynamical equations that have been derived using the operator formalism of Martin, Siggia, and Rose. (1) More importantly, it is easily extended to a broad, new class of nonlinear dynamical equations with random coefficients. In particular the equations of motion for the correlation and response functions are determined for classical systems with non-Gaussian initial conditions, multiplicative random forces, and norlinear interactions which are nonlocal in time. These results have applications in the calculation of particle motion in stochastic magnetic fields, in the solution of stochastic wave equations, and in the description of electromagnetic Vlasov turbulence. As an illustration of the new results for nonlocal interactions, the electroragnetic dispersion tensor is calculated to first order in

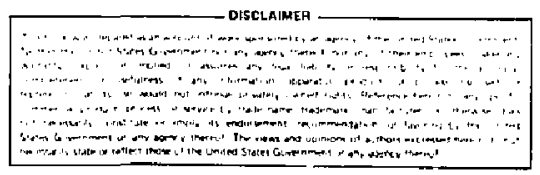


renormalized perturbation theory.

KEY WORDS: classical statistical dyramics; stochastic differential equations; Eunctional integral formalism; Schwinger equations; Dyson equations; turbulence; non. linear electromagnetic dispersion tensor. 
1. INTRODUCTION

The first satisfactory theory for the calculation of the statistical properties of classical dynamical systems was developed by Martin, Siggia, and Rose (1) (MSR), who constructed a Heisenberg operator theory which parallels the sehwinger formalism ${ }^{(2)}$ of quantum field theory. They derive closed equations for the statistical correlation and response Functions, which can be used as a starting point for systematic perturbation theories. Considerable effort has been expended in refining and extending this operator theory. $(3-6)$

Although functional integral techniques have a long and successful history in quantum theory and equilibrium statistical mechanics (7) they have only recently been exploited in the study of classical statistical dynamics. (8) De Dominicis $^{(9)}$ and Janssen (10) have shown that the equations of notion for the correlation and response functions given by MSR can also be derived from a functional integral solution to the underlying stochastic sifferential equations.

This functional integral method is analogous to Feynman's path integral formalism for quantum theory. (7) It is a more natural and elegant approach to the statistical dynamics of classical systems. Whereas MSR are reguired to introduce, ad hoc, an unphysical operator which is "conjugate" to the classical random field, the analog of this cperator appears naturally in the functional integral method. 
We have extended the functional integral approach to a much broader class of nonlinear stochastic differential equations. Because of the ad hoc nature of the original MSR formalism, it has proven difficult to extend the original method to non-Gaussian initial conditions ${ }^{(6)}$ and multiplicative random forces ${ }^{(3-4)}$ and impossible to extend it to nonlocal interactions. However, al' of these extensions are straightforward in our functional integral formalism.

In Section 2. A we establish our notation and define a class of stochastic differential equations which includes many problems of physical interest; and the operator formalism of MSR is reviewed in Section 2. 3 to introduce the fundamental ideas involved in the description of classical statistical dynamics. In section 2.C we develop our functional integral formalism which provides a formal statistical solution for the entire class of dynamical equations defined in section 2.A. This is the primary contribution of this paper.

Our formalisn encompasses all previous work on stochastic differential equations with arbitrary random initial conditions and local forces; and it provides new results for forces and interactions which are nonlocal in time. In section 3. A we recover neker's (6) results for the corrections due to non-Gaussian initial conditions. The equations of motion for the correlation and response functions for a dynarical system with a multiplicative random force are derived in section 3.B. These results have also been derived by Phytliian. They differ from the equations of Deker and Haake (3) in that the statistics of the random force are decoupled from the statistics of the random field. This separation of the statistical averages has prac- 
tical advantages. We further note that the results of Deker and Haake ${ }^{(3)}$ are also easily derived using a slight modification of our techniques. Thus the functional integral approach serves to unify the different results for this problem.

Finally, in section $3 . \mathrm{C}$ we derive the statistical equations for nonlinear dynamical systems with non local interactions. These new results provide a complete formal description of the statistical dynamics of an important class of stochastic differential equations. We then use the equations for the correlation and response functions to derive the electromagnetic dispersion tensor in lowest order renormalized perturbation theory (Direct Interaction Approximation). This p-ovides a practical application of our formal results. 
2. THE OPERATOR AND FUNCTIONAL INTEGRAL THEORIES OF CLASSICAL STATISTICAI DYNAMICS

A. Stochastic Differential Equations.

Consider the class of stochastic differential equations which car be written in the following generic form:

$$
\begin{aligned}
a_{t_{1}} \psi(1)=U_{1}(1) & +U_{2}(12) \psi(2)+U_{3}(123) \psi(2) \psi(3) \\
& +\ldots+U_{n}(1 \ldots n) \psi(1) \ldots \psi(n) \\
& +\delta\left(t_{1}-t_{0}\right) \psi_{0}(1)
\end{aligned}
$$

where $\psi(1)$ is in general a real, multicomponent classical field defined on $\mathbb{R}^{\mathrm{d}+1} \times \mathbb{Z}^{\mathrm{M}}$ which has a jump discontinuity at $t_{1}=t_{0}: \psi(1) \equiv H\left(t_{1}-t_{0}\right) \psi(1)$. The index $1 \equiv\left(t_{1}, x_{1} \ldots x_{d}, n_{1} \ldots n_{m}\right)=\left(t_{1}, l\right)$ represents the time, space, and other variables and internal indices which are arguments of the field $\psi(1) ;$ and sumation and integration over repeated indices is assumed. Moreover, the "forces" and interactions $U_{i}(1 \ldots i)=\bar{U}_{i}(1 \ldots i)+\tilde{U}_{i}(1 \ldots i)$ are integrodifferential operators which can be decomposed into a deterministic plece $\bar{U}_{i}(1 \ldots i)$ and a random piece $\tilde{\mathrm{U}}_{i}(1 \ldots i)$ with known statistics. The interactions are also required to be causal. In other words, if $\mathrm{J}_{\mathrm{n}}(1 \ldots \mathrm{n})$ involves time integrations, the integrals can only range from $t_{0}$ to $t_{1}$. Finally, the initial condition will generally consist of a deterministic and a random piece: $\psi_{0}=\bar{\psi}_{0}+\Psi_{0}$.

The fundamental statistical quantities are the mean field $\langle\psi(1)\rangle$, the fluctuation function or cumulant function 
$\langle\psi(1) \psi(2)\rangle_{C} \equiv\langle\psi(1) \psi(2)\rangle-\langle\psi(1)\rangle\langle\psi(2)\rangle$ and the averaged response function to infinitesimal external perturbations $R(12)=\left.\left\langle\frac{\delta \psi(1)}{\delta \bar{U}(2)}\right\rangle\right|_{\bar{U}(2)=0^{*}}$ Here the brackets $\langle\ldots\rangle$ will be used to indicate averages over all random elements in the problem.

We will develop a complete formal description of the statistical dynamics for this general class of stochastic differential equations. Since many interesting physical prcblems can be cast in this form, their formal solution will constitute special cases of our results.

Some important problems which lead to stochastic differential equations of this type are discussed below for il.lustration.

(i) Navier-stokes Turbulence with a Random stirring Force The Navier-Stokes equation for a randomly stirred, incompressible fluid is:

$$
\partial_{t} v+\underset{\approx}{P}: \underline{v} \cdot \nabla \mathrm{X}=v \nabla^{2} \mathrm{v}+\underline{\mathrm{f}}
$$

where $p_{t}$ is the transverse projection operator, $v$ is the viscosity, and $f$ is a transverse, random stirring force with known statistics. This is an example of a problem with a linear random force. The correspondence with (2.1) follows by identifying:

$$
\begin{aligned}
& \psi(1) \equiv v_{i}\left(t_{1}, x_{1}\right) H\left(t_{1}-t_{0}\right) \quad i_{1}=1,2,3 \\
& \tilde{U}_{1}(1) \equiv f_{i_{1}}\left(t_{1}, x_{1}\right)
\end{aligned}
$$




$$
\bar{U}_{2}(12) \equiv v \nabla^{2} \cdot \delta(1-2)
$$

$\bar{U}_{3}(123) \equiv-\left[P_{t}\right]_{i_{1}} i_{3} \nabla_{i_{2}}\left(\underline{x}_{3}\right) \cdot \delta\left(t_{1}-t_{2}\right) \delta\left(\underline{x}_{1}-\underline{x}_{2}\right) \delta(1-3)$

\section{(ii) Particle Motion in Stochastic Magnetic Fields} Krommes, Kleva, and Oberman (13) have derived a stochastic differential equation for the evolution of the phase space density $P(x, v, t)$ of charged particles moving along magnetic field lines. The magnetic field is assumed to be primarily in the 2 direction with weak shear in the $y$ direction and a small random component $b(x, t)$ in the $x$ direction. Their result for $x \ll L_{s}$ is

$$
\frac{\partial P}{i t}+v\left[\frac{\partial}{\partial z}+\frac{x}{L_{s}} \frac{\partial}{\partial Y}\right] P-D \frac{\partial}{\partial y}^{2}-c\{v\} P+v b \frac{\partial}{\partial r} Y=\delta\left(t-t_{0}\right) P_{0}
$$

where $v$ is the particie velocity along the field ines, $L_{s}$ is the shear length, $D$ is the classical pexpendicular diffusion coefficient due to particle collisions, and $C\{v\}$ is a collision operator in velocity space.

Eguation (2.4) is an example of a stochastic differential equation with a multiplicative random force which can be written in the form of (2.1) by identifying:

$$
\begin{aligned}
\psi(1) & \equiv P\left(t_{1}, \mathrm{x}_{1}, \mathrm{v}_{1}\right) H\left(\mathrm{t}_{1}-t_{0}\right) \\
\mathrm{U}_{1}(1) & \equiv 0 \\
\overline{\mathrm{U}}_{2}(12) & \equiv-\left\{\mathrm{v}_{1}\left[\frac{\partial}{\partial \mathrm{z}_{2}}+\frac{\mathrm{x}_{1}}{\mathrm{~L}_{\mathrm{s}}} \frac{\partial}{\partial \mathrm{y}_{2}}\right]-D \frac{\partial^{2}}{\partial \mathrm{y}_{2}^{2}}-\mathrm{C}\left\{\mathrm{v}_{2}\right\}\right\} \cdot \delta(1-2) \\
\widetilde{\mathrm{U}}_{2}(12) & \equiv-v_{1} \mathrm{~b}(1) \frac{\partial}{\partial \mathrm{x}_{2}} \cdot \delta(1-2) .
\end{aligned}
$$


(iii) Stochastic Wave Equation

The propagation of waves in random media is described by stochastic wave equations of the form

$$
\frac{\partial^{2} \phi}{\partial t^{2}}=b \nabla^{2} \phi
$$

where $b$ is a random variable with known statistics. If we integrate (2.6) once with respect to time using cauchy initial conditions $\phi\left(t_{0}\right)=\phi_{0}$ and $\frac{\partial \phi}{\partial t}\left(t_{0}\right)=\frac{\partial \phi_{0}}{\partial t}$, then we get a stochastic differential equation with a nonlocal interaction

$$
\frac{\partial \phi}{\partial t}=\int_{t_{1}}^{t} \partial t^{\prime} b \nabla^{2} \phi+\frac{\partial \phi}{\partial t} 0 \text {. }
$$

This equation can then be written in the form of (2.1) by identifying

$$
\begin{aligned}
& \psi(1)=\phi\left(t_{1}, x_{1}\right) H\left(t_{1}-t_{0}\right) \\
& U_{1}(1)=\frac{\partial \phi}{\partial t}\left(x_{1}\right) \\
& U_{2}(12)=b\left(t_{2}, x_{2}\right) \nabla^{2} H\left(t_{1}-t_{2}\right) \delta\left(x_{1}-x_{2}\right) .
\end{aligned}
$$

(iv) Electromagnetic Vlasov Turbulence

The Vlasov-Maxwell equations describe the collisionless evolution of distributions of charged particles $f_{s}(\underset{\sim}{x}, t, t)$ and their associated electric and magnetic fields: 


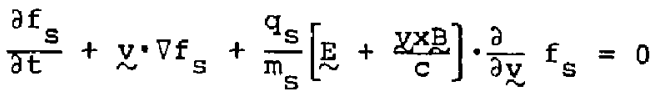

$$
\begin{aligned}
& \frac{\partial \mathrm{E}}{\partial t}-\mathbf{c} \nabla \times \mathrm{P} \sim+4 \pi r q_{s} \int a^{3} v f_{s} \mathrm{v}=0 \\
& \frac{\partial R}{\partial t}+c \nabla \times E=0
\end{aligned}
$$

where $s$ is the charged particle species index, $q_{s}$ is the charge, and $\mathrm{m}_{\mathrm{s}}$ is the mass. Statistics enters the problem either through the assumption of 1) random initial conditions or 2) some implicit randomness in the distribution functions which requires ensemble averages to be taken to define quantitjes of physical interest (i.e., ranciom phases). This problem can be cast into the form of (2.1) in two different ways. First, $\psi(I)$ can be defined to be a vector field with $\mathrm{N}+6$ components where $\mathrm{N}$ is the number of charged particle species. The remaining 6 components arise from the vector electric and magnetic fields. Alternatively, (2.10) and (2.11) can be used to solve for $\underset{\sim}{\mathrm{E}}$ and $\underset{\sim}{\mathrm{B}}$ in terms of the particle distribution functions. Then $\psi(1)$ represents only the $\mathrm{N}$ particle distributions. Although the second method introduces nonlocal interactions through retardation effects, it reduces the number of components of the vector field $\psi$. This reduction has computational advantages in the usual cases wtere $\mathrm{N}=1$ or 2 .

Here we will follow the second approach. We solve (2.10) and 
(2.11) in the usual way by introducing the scalar and vector potentials $A^{0}$ and $\mathbb{A}^{(16)}$. The results are

$$
\begin{aligned}
& \underset{\sim}{B}\left(x_{1}, t_{1}\right) \equiv \underset{\sim}{B}(12) \psi(2)=\nabla_{1} x A(12) \psi(2) \\
& \underset{\sim}{E}\left(x_{1}, t_{1}\right) \equiv E(12) \psi(2)=-\nabla_{1} A^{0}(12) \psi(2)-\frac{1}{c} \frac{\partial}{\partial t_{1}} A(12) \psi(2)
\end{aligned}
$$

where we have defined $\psi(1) \equiv f\left(t_{1}, x_{1}, \underline{y}_{1}, s_{1}\right)$. The definitions of the four potential operators depend on the choice of gauge. In the coulomb gauge $\nabla \cdot A=0$ for example, we have a retarded vector potential and an "instantaneous" scalar potential :

$$
\begin{aligned}
& A\left(x_{1}, t_{1}\right) \equiv A(12) \psi(2)
\end{aligned}
$$

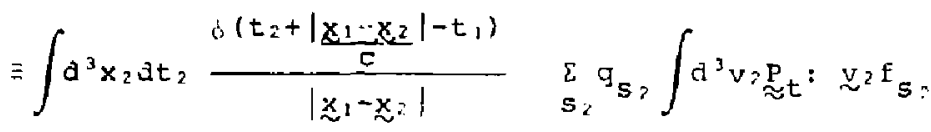

$$
\begin{aligned}
& A^{0}\left(x_{1}, t_{1}\right) \equiv A^{2}(12) \psi(2) \\
& \equiv \int d^{3} x_{2} d t_{2} \frac{\delta\left(t_{2}-t_{1}\right)}{\left|x_{1}-x_{2}\right|} \quad \sum q_{s_{2}} \int d^{3} v_{2} f_{s_{2}}
\end{aligned}
$$

where $\mathrm{P}_{\mathrm{t}}$ is the transverse projection operator.

The retarded four-potential gives rise tu nonlinear interactions which are nonlocal in time. Since the potentials are retarded the interactions are also causal. The evolution of $(1)$ depenas only on the past, not on the future.

The correspondence of equations (2.9), (2.10), and (2.11) with 
equation (2.1) is completed by identifying

$$
\begin{aligned}
& U_{1}(1) \equiv 0 \\
& \overline{\mathrm{U}}_{2}(12) \equiv-\mathrm{V}_{1} \cdot \nabla_{\mathrm{x}_{1}} \cdot \delta(1-2) \\
& \overline{\mathrm{u}}_{3}(123) \equiv-\frac{\mathrm{q}_{1}}{\mathrm{~m}_{1}}\left[\underset{\sim}{E}(12)+\frac{\mathrm{v}_{1} \times}{G} \mathrm{~B}(12)\right] \cdot \frac{\partial}{\partial \overline{\mathrm{v}}_{1}} \cdot \delta(1-3) \\
& \equiv-L(1 ?) \cdot \frac{\partial}{\partial V^{\prime}} \cdot \delta(1-?)
\end{aligned}
$$

where $L(12)$ is the Lorentz force operator.

B. The Operator Formalism of Martin, Siggia, and Rose.

In order to describe the statistical properties of a classical dynamical system 'which is governed by a stochastic differential equation, we need a theory for the calculation of the correlation functions and response functions (avereged Green's functions). If we naively average stochastic differeni-ial equations such as (2.1) with resfect to random forces and interactions, randum initial corditions, or an ensemble of realizations, we arrive at an equation for the evolution of $\langle\psi(1)\rangle$. Unfortunately, the dyramics of $\langle\psi(1)\rangle$ will depend in general upon higher order correlation functions $\left\langle u_{n}(1 \ldots n) \psi(2) \ldots \psi(n)\right\rangle$ due to the nonlinear interactions $\bar{U}_{n}(1 \ldots n), n \geq 3$ and $\widetilde{U}_{n}(1 \ldots n), n \geq 2$. The evolution of these higher order correlation functions depends in turn upon still higher order correlations. The resulting hierarchy of equations can only be closed by some truncation procedure. (17)

Martin, Siggia, anc Rose ${ }^{(1)}$ developed the first satisfactory method for overcoming this ffficulty. They succeed in deriving 
closed, exact equations for the evolution of the first few statistical correlation and response functions. Although these exact equations are complicated, they provide a starting point for a renormalized perturbation theory. In addition, the fundamental objects of the theory-the mean field $\langle\psi(1)\rangle$, the fluctuation function $\langle\psi(1) \psi(2)\rangle_{C}$, and the response function $R(12)$ to infinitesimal perturbations - are the physical "observables" of greatest interest,

In order to take advantage of the powertul methods of quantum field theory, MSR treat the classical field $\psi(1)$ a'; a Heisenberg operator. The classical correlation functions are then defined to be "vacuum" expectation values of time-ordered products of these operators. The important contribution of MSR was the ad hoc introduction of a complex zäjoint operator $\hat{\psi}(t, 1)$ which does not commute with $\psi(t, 2):$

$$
[\hat{\psi}(t, \underline{2}), \psi(t, 2)]=\delta(\underline{\sim}-2) .
$$

$\hat{\psi}$ is defined, moreover, such that the time-ordered vacuum expectations of products of operators vanish whenever $\hat{\psi}$ is the latest operator: $\langle\hat{\psi}(1) \hat{\psi}(2) \hat{\psi}(3) \ldots\rangle_{+}=0$ if $t_{1}>t_{2}, t_{3} \ldots$. In particular $\langle\tilde{\psi}(1)\rangle=0$.

The time-ordered expectation value of $\psi(1)$ and $\hat{\psi}(2)$ gives the averaged response function (18) 


$$
R(12)=\langle\psi(1) \hat{\psi}(2)\rangle_{+}
$$

and the definition of time-ordered expectations of products of the operators $\psi$ and $\hat{\psi}$ insures the causality of $R$. In fact, all statistical quantities of interest are determined by expectations of imeordered products of the operators $\psi$ and $\hat{\psi}$. In the theory of quantum fields these expectations are the Green's functions.

The adjoint operator also makes it possible to construct a damiltoniar. $H$ which generates the equations of motion for the operators $\psi(i)$ and $\hat{\psi}(1)$. Let $\underset{\sim}{\Phi}(1) \equiv\left(\begin{array}{l}\psi(1) \\ \psi(1)\end{array}\right)$, then

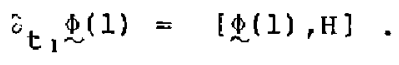

This approach is only applicable to dynamic equations (2.1) with local, deterministic forces and interactions; then:

$$
H \equiv \hat{\psi}(\overline{\mathrm{I}})\left[\overline{\mathrm{U}}(\overline{\mathrm{I}})+\overline{\mathrm{U}}_{2}(\overline{\mathrm{I}} 2) \ldots \overline{\mathrm{L}}_{n}{ }_{2}(\overline{\mathrm{I}} \ldots \mathrm{n}) \psi(\overline{\mathrm{a}}) \ldots \psi(\mathrm{n})\right]
$$

Equation (2.17) has the same form as the equations of quantum field theory. We can, therefore, apply the schwinger functional formalism $^{(2)}$ to derive closed equations for the exact Green's functions. The first step is to define a generating functional

$$
\bar{z}(\underset{\sim}{n}\} \equiv\langle\exp \{\underset{\sim}{n}(1) \cdot \underset{\sim}{\Phi}(1)\}\rangle_{+} \equiv\langle 1\rangle_{+}^{n}
$$

The various Green's functions are determined by evaluating functional

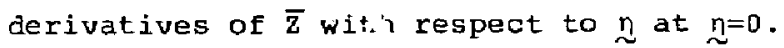

It is convenient to work with the connected Green's functions which are generated by $\overline{\mathrm{F}}\{\underset{\sim}{\eta}\} \equiv 1: \overline{\bar{z}}\{\underset{\sim}{\eta}\}$. The schwinger equations for the evolution of the one point connected Gren's functions 


$$
G_{1}^{\eta}(1) \equiv \frac{\partial \bar{F}}{\partial \eta(1)} \equiv\left\langle\frac{\Phi(1)}{\bar{z}}\right\rangle_{+}^{n}
$$

are easily derived from (? 17 )

$$
\begin{aligned}
& \partial_{t_{1}} G_{1}^{\prime \prime}(1)=\left[\langle\dot{\Phi}(1)\rangle_{+}^{n}+\langle\Phi(1) \exp (\underset{\sim}{\sim}(1) \cdot \Phi(1))\rangle_{+}\right] \frac{1}{\bar{z}} \\
& =\left\langle\frac{([(I), H]}{\bar{z}}\right\rangle_{+}^{\eta}+i \sigma_{2} \sim_{\sim}(I)
\end{aligned}
$$

where $\sigma_{2}=\left(\begin{array}{rr}0 & -i \\ i & 0\end{array}\right)$.

The two-point connected Green's functions

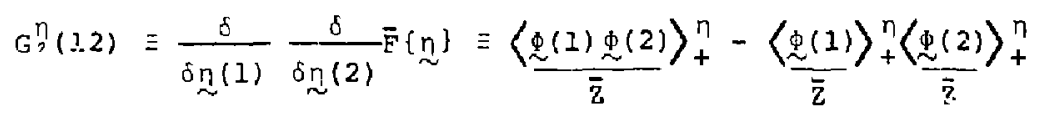

are just the fluctuation and response functions. Their evolution is destribed $t_{y}$ the Dyson equations which result from the functional differentiation of $\{2.19\}$ with respect to $\mathrm{D}(12)$ :

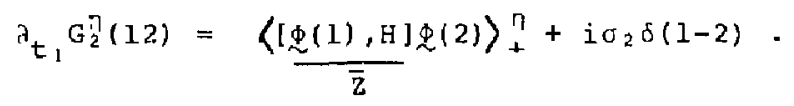

The schwinger and Dyson equations (2.19) and (2.20) are the first in a hierarchy of equations. However, this hierarchy can be formally closed, exactly, by the following procedure. First, the Green's functions $\langle\Phi(2) \ldots \Phi(n)\rangle_{+}^{n}$ on the r.h.s. of $(2.19)$ and $(2.20)$ are writter in terms of the connected Green's functions. The connected Green's functions $G_{n}^{n}(1 \ldots n)$ are in turn written in terms of two-point connected Green's functions $G_{2}^{\eta}$ and the 1-particle irreducible vertex functions $r_{n}(1 \ldots n)$. The generating functional for these vertex functious results from a Legendre transform of $\overline{\mathrm{F}}$ : 


$$
\Gamma\left\{G_{1}^{n}\right\} \equiv \bar{F}\{n\}-\underset{\sim}{n}(1) \cdot G_{1}^{n}(1)
$$

The vertex functions are then given by functional derivatives of $\Gamma\left\{G_{1}^{n}\right\}$ with respect to $C_{1}^{n}$ evaluated at $G_{1}$ for $\eta=0$. Since

$$
\left.\Gamma_{2}(12) \equiv \frac{\delta}{\delta G_{1}^{\eta}(1)} \frac{\delta}{\delta G_{1}^{\eta}(2)} \Gamma\left[G_{1}^{\eta}\right]\right|_{\eta=0}=-G_{2}^{-1}(12)
$$

we can write any vertex function as (19):

$$
\Gamma_{n}(1 \ldots n) \equiv \frac{-\delta}{\delta G_{1}(3)} \cdots \frac{\delta}{\delta G_{1}(n)} G_{2}^{-1}(12) .
$$

Therefore, every Green's function on the r.h.s. of (2.19) and (2.20) can be formally expressed in terms of the two-point connected Green's functions and the vertex functions which are given by $(2,22)$ as furictional derivatives of $\vec{G}_{2}^{-1}$ with respect to $G_{1}$. cunseguently, in the limic $n \rightarrow 0$, the schwinger and Dyson equations can be written as formalizy closed functional equations for the exact one-point and two-point connected Green's functions $G_{1}$ and $G_{2}$.

Ir their original paper MSR were primayily concerned with dynamical systems with a quadratic, deterministic interaction. The statistics appear through averages with respect to Gaussian initial conditions or an ensemble of realizations. This corresponds to a stachastic differentici equation of the form of (2.1) with: 


$$
\begin{aligned}
U_{1}(1) & =\bar{U}_{2}(1) \\
U_{2}(12) & =\bar{U}_{2}(12) \\
U_{3}(123) & =\bar{U}_{3}(123) \\
U_{n}(1 \ldots n) & =0, n>3
\end{aligned}
$$

The closed operator equations (2.19) and (2.20) tor che statistical dynamics of these systems are written compactly in MSR's notation as

$$
\begin{gathered}
{\left[G_{2}^{0}\right]^{-1} G_{1}=\frac{1}{2} \gamma_{3} G_{1}^{2}+\frac{1}{2} \gamma_{3} G_{2}+\gamma_{1}} \\
G_{2}^{-1}=\left[G_{2}^{0}\right]^{-1}-Y_{3} G_{1}-\Sigma
\end{gathered}
$$

where we have taken $\sim_{n}^{n}=0 . \quad\left[G_{2}^{0}\right]^{-1}(12) \equiv-j \cdot \sigma_{2} a_{t_{1}} \delta\left(t_{1}-t_{2}\right)-Y_{2}(12)$ is the "bare" two-point propagator; and the resonance broadening term $\Sigma$ is defined by

$$
\Sigma \equiv \frac{1}{2} \gamma_{3} G_{2} G_{2} \Gamma_{3}
$$

Finally, the three-point vertex function is given by (2.24) and (2.22)

$$
\Gamma_{3}=Y_{3}+\frac{\delta \Sigma}{\delta G_{2}} G_{2} G_{2} \Gamma_{3}
$$

where we used the chain rule to write: $\frac{\varepsilon}{\delta G_{1}}=\frac{\delta G_{2}}{\delta G_{1}} \frac{\delta}{\delta G_{2}}=G_{2} G_{2} \Gamma_{3} \frac{\delta}{\delta G_{2}}$.

MSR also comider a system stirred by a random Gaussian force $\tilde{U}_{1}(1)$. Although their method does not provide a airect means 
of determining the statistical dynamics, they note that a Gaussian random force with vanishing mean can be represented by a deterministic correction to $\mathrm{H}$ of the form $\hat{\mathrm{U}}_{2}(12) \hat{\psi}(1) \hat{\psi}(2)$ where $\hat{U}_{.}(2) \equiv\langle\langle\tilde{u}(1) \tilde{U}(2)\rangle\rangle$ is the cumulant average of the random force. The calculation of closed dynamical equations then proceeds as before.

Equations $(2.23)-(2.26)$ can be solved approximately by systematical.ly expanding the exact equations in some small parameter. This is much more satisfying than the conventional perturbation procedure, in which "small" corrections are added to approximate equations in the hope of improving the approximation. Additional advantages of this approach lie in the fact that the physical symmetries of the exact solution are manifest in the exic: equations of motion but they may be absent in a method which starts from approximate equations.

Althoigh it is clear that a complete formal theory for the statistical dynamics of classical systems has many important advantages, the original method developed $\$$ MSR is Ijmited to a restricted class oŕ stochastic differential equations. Specifically, the only random processes treated had deterministic interactions, linear random forces with Gaussian statistics, and Gaussian initial conditions Deker and Hake ${ }^{(3)}$ and Phythian ${ }^{(4)}$ have extended the MSR formalism to multiplicative random forces and very recently Deker ${ }^{(6)}$ has refined and extended it to non-Gaussian random initiai conditions, forces, and interactions.

Because of the ad hoc origin of the MSR formalism the extensions 
to more complicated statistical systems have each required a separate treatment. The MSR theory does not provide a general formalisı, which naturally generates the statistical equations of motion for the entire class of stochastic differential equations described by (2.1). In fact, nonlocal interactions have proven to be intractable for any extension of the MSR approach.

C. Functional Integral Formalism

An alternative approach to the description of classical dynamical systems was introduced by Janssen (10) and De Dominicis. (9) They discoverec that a functional integral formalism, analogous to Feynman's theory ${ }^{(7)}$ for quantum mechanics, provides a very natural and elegant derivation of MSR's results for quadratic deterministic interactions and Gaussian random forces. Phythian (11) has pursued the functional integral formalism further and shown that the statistical equations of motion for miltiplicative random forces are also easily derived with tis approach.

We will. show that the functional integral method provides a complete formal description of the statistical dynamics for the entire class of stochastic djefferential equations defined by equation (2.1). This is the primary contribution of this paper. Our work unifies all previous results and provides the formal solution to several new problems of physical interest.

In this section we develop the functional integral theory and demonstrate how the MSR equations can be easily recovered. The specific results for non-Gaussian initial conditions, multiplicative random forces, and nonlocal interactions are discussed in later 
sections.

Consider a multicomponent classical field $\psi(1)$ which satisfies a stochastic differential equation of the type described by Equation (2.1). In order to define a functional integral we coarse grain the multidimensional space spanned by the time, position, and other continuous arguments of $\psi(1)$. The coarse-graining procedure defines a lattice which partitions the $\mathrm{d}+1$ aimensional space into small volumes of size $\varepsilon^{d+1}$. The index 1 becomes a discrete index which labels the vertices on the lattice, ard the stochastic nifferential equation is transformed into a difference equation.

The functional integral is formally defined to be the multiple integral over the range of $\psi(i)$ at every lattice point in the Iimit $E \rightarrow 0$

$$
\int D[\psi] \ldots \equiv \lim _{\varepsilon \rightarrow 0} \prod_{i \in \Lambda} d+1 d \psi(i) \ldots
$$

where $\Lambda^{d+l}$ denotes the set of vertices on the lattice. Although the general mathernatical theory for these infinite multiple integrals is incomplete, they have nevertheless proven useful in generating significant results. Consequently, we will not digress to discuss this technical pcint but will refer tic reader to the literature

For the purpose of illustration consider a system of one degree of freedom. If $\psi(1)$ depends only on time, for $: 0 \leqslant \tau_{1} \leqslant t$, then the interval $\left[t_{0, t]}\right.$ can be divided : $: 0 \mathrm{~N}$ segments of length $\varepsilon$ and Equation '2.1) can be discretized in many different ways. For 
example:

$$
\begin{aligned}
& \frac{\psi\left(t_{i}\right)-\psi\left(t_{i-1}\right)}{\varepsilon}=a_{1} U_{1}\left(t_{i}\right)+\beta_{1} U_{i}\left(t_{i-1}\right)+\ldots \\
& \quad+\left[\alpha_{n} U_{n}\left(t_{i}, t_{2} \ldots t_{n}\right)+\beta_{n} U_{n}\left(t_{i-1}, t_{2} \ldots t_{n}\right)\right] \psi(2) \ldots \psi(n)+\frac{\delta_{i 0}}{E} \psi_{0}
\end{aligned}
$$

where $\alpha_{i}+\beta_{i}=1$. Still other disctetization schemes can be devised; however, as $\epsilon \rightarrow 0$, all of these should be equivalent. (21)

The functional integral is simply

$$
\int D[\psi] \ldots \equiv \lim _{\varepsilon \rightarrow 0} \prod_{i=0}^{N} \int_{0} d \psi(i) \ldots
$$

The generalization of these definitions to systems with many degrees of freedom is strightforward

In developing our formalism we follow the approach of Jouvet and Phythian (23) and consider first the formal functional integral represent-jion of the solutions of deterministic equations of motion. Consider any functional $F(\psi)$ of the classical field $\psi(1)$ which satisfies a dynamical equation of the form of (2.1). For the moment we will treat all stochastic forces and interactions as if they were deterministic and write

$$
F\{\psi\} \equiv \int D\left[\psi^{\prime}\right] \delta\left(\psi^{\prime}-\psi\right) F\left\{\psi^{\prime}\right\}
$$

where $\psi$ is the unique solution to the differential equation and the functional $\delta$ function is defined by: $\delta\left(\psi^{\prime}-\psi\right)=\underset{E \rightarrow 0}{\lim } \underset{i \in \Lambda^{d+1}}{d\left(\psi^{\prime}(i)-\psi(i)\right)}$. 
Since $\psi$ is determined by an algebraic difference equation like $(2.28)$ we can make a convenient change of coordinates:

$$
\begin{aligned}
& F\{\psi\}=\int D\left[\psi^{\prime}\right] \delta\left[\psi^{\prime}(1)-U_{1}(1)-U_{2}(12) \psi^{\prime}(2)+\ldots\right. \\
& \left.+U_{n}(1, \ldots n) \psi^{\prime}(2) \ldots \psi^{\prime}(n)+\delta\left(t_{1}-t_{0}\right) \psi_{0}\left(\frac{1}{2}\right)\right] J\left(\psi^{\prime}\right) H^{\prime}\left\{\psi^{\prime}\right\}
\end{aligned}
$$

where $J\left(\psi^{\prime}\right)$ is the Jacobian that results from the coordinate change. The r.h.s. of (2.31) signifies that the integrand is non-zero only for $\psi^{\prime}$ which satisfies the discretized dynamical equation.

The explicit form of the Jacobian depends on the manner in which the dynamical equation is discretized. Since the different discretizations give the same final results, it will prove convenient to choose one such that $J=\prod_{i \in \Lambda} a+1 \frac{l}{E}$ is independent of $\psi(i)$. For the one-dimensional problem this corresponds to the requirement that $\alpha_{i}=0$ and $\beta_{i}=1$ in $(2.28)$. Although $J$ is infinite as $c \cdot 0$, this divergence will be cancelled by another divergent onstant in the final equations.

The nexr step is to repiace the $\delta$ function by its functional Fourier transform which gives

$$
\begin{gathered}
F\{\psi\}=c \int_{D} D\left[\psi^{\prime}\right] D[\hat{\psi}] \exp -\left\{\hat { \psi } ( 1 ) \left[\dot{\psi}^{\prime}(1)-U_{1}(1)-U_{2}(12) \psi^{\prime}(2)\right.\right. \\
\left.\left.-\ldots U_{n}(12 \ldots n) \psi^{\prime}(2) \ldots \psi^{\prime}(n)-\delta\left\langle t_{1}-t_{0}\right\rangle \psi_{0}\right]\right\} F\left\{\psi^{\prime}\right\}
\end{gathered}
$$

where $c=\prod_{i \in \Lambda} d+1\left[\frac{1}{2 \pi \varepsilon}\right] . \quad$ The Fourier transform $\hat{\psi}(1)$ is an ima-

ginary field. Our definition differs from Jouvet and Phythian's (23) by an explicit factor of $(-i)$. As before this formal result can be 
justified by returning to the discrete lattice and then taking $E \rightarrow 0$, where it is conventional to displace the discrete time arguments of $\hat{\psi}$ such that $(24)$

$$
\hat{\psi}(t) \psi(t) \equiv \hat{\psi}\left(t_{i}+\frac{\varepsilon}{2}\right)\left(t_{i}\right)
$$

in order to avoid time-ordering ambiquities.

By comparing (2.32) with the functional integrals which occur in field theories we can identify a Lagrangian $L$ and a Hamiltonian $H$

$$
\begin{aligned}
L & \equiv \hat{\psi}(1)\left[\dot{\psi}(1)-\mathrm{U}(1)-\mathrm{U}_{2}(12) \psi^{\prime}(2)-\ldots\right. \\
& \left.\quad-\mathrm{U}_{n}(1 \ldots n) \psi^{\prime}(2) \ldots \psi^{\prime}(n)-\delta\left(t_{1}-t_{0}\right) \psi_{0}(1)\right] \\
& \equiv \hat{\sim}(1) \dot{\psi^{\prime}}(1)-H\left\{\psi^{\prime}, \hat{\psi}\right\}
\end{aligned}
$$

which allows us to write (2.32) compactly as

$$
F\{\psi\}=c \int D\left[\psi^{\prime}\right] D[\hat{\psi}] \quad F\left\{\psi^{\prime}\right] \exp (-\Sigma) .
$$

We will see that the new field $\psi$ which occurs naturally in (2.32) is the exact analog of the non-commuting operator $\hat{\psi}$ which was introduced ac boc by MSR. In fact, it has been shown that the operator theory of MSR can be derived directly from this functional integral formalism just as the Heisenberg operator theory of quantum mechanics is a consequence of Feynman's path integral formalism. $(21,23,25)$ However, rather than emphasize the reduction to the earlier operator theory we will pursue the development of the more natural and powerful functional integral theory. Although $\hat{\psi}$ appears in $(2.32)$ simply as a fourier transform 
variable, phythian (11) has shown that it plays a crucial role in the description of dynamical systems. Consider the response of

$F\{\psi\}$ to an infinitesimal linear perturbation to the dynamical equations $U_{1}(1) \rightarrow U_{1}(1)+\xi_{1}(1)$. Then

$$
\begin{aligned}
& \mathrm{o} \mathbf{F}\{\psi\}=\mathrm{c} \int \mathrm{D}\left\{\psi^{\prime}\right] \mathrm{D}[\hat{\psi}]\left|\mathrm{e}^{-[L-\hat{\psi}(1) \zeta(1)]}-\mathrm{e}^{-l}\right| \mathrm{F}\left\{\psi^{\prime}\right\} \\
& =c \int D\left[\psi^{\prime}\right] D[\hat{\psi}] \exp (-L)\left[\hat{\psi}(1) \sum(1)+\hat{\psi}(2)^{2} \xi(1)^{2}+\ldots\right] F^{\prime} \psi^{\prime}
\end{aligned}
$$

and the linear response function is simply

$$
\left.\frac{\delta F[\psi]}{\delta \xi(1)}\right|_{\zeta(1)=0}=c \int D\left[\psi^{\prime}\right] D[\hat{\psi}] e^{-L}\left[\hat{\psi}(1) F\left[\psi^{\prime}\right\}\right],
$$

The linear response functions to many infinitesimal disturbances is in general given by

$$
\left.\frac{\delta^{n} F[\psi]}{\delta \xi_{1}\left(1^{\prime} \ldots \delta \xi_{n}(n)\right.}\right|_{\xi_{i}=0}=c \int D\left[\psi^{\prime}\right] D[\hat{\psi}] e^{-L}\left[(\hat{\psi}(1) \ldots \hat{\psi}(n)) F\left[\dot{\psi}^{\prime}\right]\right]
$$

We can now reintroduce the statistics. The functional integral representations of functionals of $\psi(2.35)$ and of the response functions (2.37) are easily averaged over the random forces, interactions, or initial conditions. For example;

$$
F\{\psi\} \equiv c \int D\left[\psi^{\prime}\right] \mathrm{D}[\hat{\psi}] \mathrm{F}\left\{\psi^{\prime}\right\}\langle\exp (-L)\rangle
$$

where all the randar elements are contained in the Lagrangian $L$. Since the statistics are generally assumed to be known, the 
average in (2.38) can be performed explicitly. This defines an averaged effective Lagrangian $\mathrm{L}$ and Hamiltonian $\mathrm{H}$

$$
\langle\exp (-L)\rangle \exp (-L) \equiv \exp -[\hat{\psi}(1) \dot{\psi}(1)-H]
$$

This averaged Lagrangian I gives rise to the statistical equations of motion.

Consider the generating functional

$$
Z\{n, \zeta\} \equiv c \int D\left[\psi^{\prime}\right] D[\hat{\psi}] e^{-L} \exp [\psi(1) n(1)+\hat{\psi}(1) \zeta(1)] .
$$

The functional $z$ contains a complete statistical description of the classical dynamical system ccrresponding to the averaged Lagrangian $L$. All of the correlation and response functions are given by functional derivatives of $z$ with respect to $\eta$ and $\zeta$.

We will formally treat $\hat{\psi}$ on ari squal footing with $\psi$ and write the averaged response functions (2.37)

$$
\begin{aligned}
\left.\frac{\delta^{n} F\{\psi\}}{\delta \xi(1) \ldots \delta \xi(n)}\right|_{\xi=0} & \equiv\langle\hat{\psi}(1) \ldots \hat{\psi}(n) F(\psi)\rangle \\
& \equiv c \int D[\psi] D[\hat{\psi}][\hat{\psi}(1) \ldots \hat{\psi}(n) F\{\psi]] \exp (-L) .
\end{aligned}
$$

Then the generating functional $z$ can be used to write the statistical average of any analytical functional $A$ of $\psi$ and $\hat{\psi}$ as

$$
\langle A\{\psi, \hat{\psi}\}\rangle=\mathrm{A}\left\{\frac{\delta}{\delta n}, \frac{\delta}{\delta \zeta}\right\} \mathrm{Z}\{\eta, \zeta\} .
$$

If the functional $A\{\psi, \hat{\psi}\}$ depends on time for $t \in\left[t_{0}, T\right]$, then the res- 
ponse to perturbations at times $t_{1}>T$ vanishes. This ensures the causality of the response functions which implies in particular tinat

$$
\langle\hat{\psi}: 1\rangle \ldots\rangle=0
$$

if $t_{1}$ is the latest time in the average.

In general the functional integral representation for the generating functional $(2.40)$ will be too complex for practical calculftions of statistical quantities. ${ }^{1}$ However, the equations of evolution for the statistical correlation and response functions can be easily obtained. Since $\mathrm{L}=\hat{\psi}(1) \dot{\psi}(1)-\mathrm{H}$ the formal schwinger equations for the evolution of $\langle\psi(1)\rangle$ and $\langle\hat{\psi}(1)\rangle$ are derived by a functional integration by parts. Using the identities

$$
\int D[\psi] D[\hat{\psi}]\left(\begin{array}{c}
\frac{\delta}{\delta \hat{\psi}(1)} \\
\frac{\delta}{\delta \psi(I)}
\end{array}\right) \exp [\eta(1) \psi(1)+\zeta(1) \hat{\psi}(1)-L]=0
$$

we get

$$
\begin{array}{r}
\frac{\langle\dot{\psi}(1)\rangle}{\mathrm{z}}-\frac{1}{\mathrm{z}}\left(\frac{\delta}{\delta \hat{\psi}(1)} H\right)-\zeta(1)=0 \\
-\frac{\langle\dot{\dot{\psi}(1)}\rangle}{\mathrm{z}}-\frac{1}{\mathrm{z}}\left(\frac{\delta}{\delta \psi(1)} \mathrm{H}\right\rangle-\eta(1)=0
\end{array}
$$

where $H$ is explicitly determined by

\footnotetext{
${ }^{1}$ Various approximate techniques have been developed in quantum field theory for the direct evaluation of the generating functional. These include saddle point methods, variational principles (7), and the Renormalization Group(19). Using the functionai jntegral formalism these powerful tools can also be applied to problems in classical statistical dynamics.
} 


$$
\begin{aligned}
H \equiv \ln \left\langle\operatorname { e x p } \hat { \psi } ( 1 ) \left[ U_{1}(1)+U_{2}(12) \psi(2)\right.\right. & +\ldots+U_{n}(1 \ldots n) \psi(2) \ldots \psi(n) \\
& \left.\left.+\delta\left(t_{1}-t_{0}\right) \psi_{0}(1)\right]\right\rangle \cdot(2.4
\end{aligned}
$$

The principal statistical quantities of physical interest are the fluctuation function

$$
\langle\psi(1) \psi(2)\rangle_{c} \equiv\langle\psi(1\rangle \psi(2)\rangle-\langle\psi(1)\rangle\langle\psi(2)\rangle \quad
$$

and the averaged response function

$$
R(12) \equiv\langle\psi(1) \hat{\psi}(2)\rangle_{\mathrm{c}} \equiv\langle\psi(1) \hat{\psi}(2)\rangle .
$$

Note that the divergent constant $c$ no longe: appears in these physical quantities. If the fluctuation and averaged response functions are rewritten as

$$
\begin{aligned}
& \left.\langle\psi(1) \psi(2)\rangle_{c} \equiv \frac{\delta}{\delta n(2)} \frac{\langle\psi(1)}{2}\right\rangle \\
& \left.\langle\psi(1) \hat{\psi}(2)\rangle_{c} \equiv \frac{\delta}{\delta \zeta(2)} \frac{\langle\psi(1)}{2}\right\rangle
\end{aligned}
$$

then the $c$ in the numerator is cancelled by the $c$ in the denominator. Mcreover, a causal response function is assured since $\frac{s}{\delta \zeta(2)}\langle\psi(1)\rangle=0$ for $t_{2}>t_{1}$.

The Dyson equations for the fluctuation and averaged response functions follow from (2.44) by functional d.rferentiation with respect to $\eta(2)$ and $\zeta(2)$

$$
\langle\dot{\psi}(1) \psi(2)\rangle_{C}-\left[\left\langle\psi(2) \frac{\delta}{\delta \hat{\psi}(1)} H\right\rangle-\langle\psi(2)\rangle\left\langle\frac{\delta}{\delta \hat{\psi}(1)} H\right\rangle\right]=0
$$




$$
\langle\dot{\psi}(1) \hat{\psi}(2)\rangle_{c}-\left[\left\langle\hat{\psi}(2) \frac{\delta}{\delta \hat{\psi}(1)} H\right\rangle-\langle\hat{\psi}(2)\rangle\left\langle\frac{\delta}{\delta \hat{\psi}(1)} H\right\rangle\right]=\delta(1-2)
$$

Then the system of statistical equations $(2.44)-(2.48)$ can be formally closed, using the same procedure as in the MSR formalism. First, the n-point field averages are expressed in terms of the connected Green's functions which are generated by $\overline{\mathrm{F}}\left\{\eta_{0}, \xi\right\} \equiv \ln z\left\{\eta_{0}, r_{n}\right\}$. Then these connected Green's furctions are written in terms of the two-point connected Green's functions and the vertex functions $\Gamma_{n}(1 \ldots n)$ which are generated by the Legendre transform of $\bar{F}$. Since all of the vertex functions are given in terms of functional derivatives of two-point functions with respect to one-point functions as in Equation (2.22), the set of Schwinger and Dyson equations forms a closed set of exact statistical equations for the mean fields, Eluctuation functions, and response functions.

After these transformations, Equations (2.44)-(2.48) represent a complete description of the statistical dynamics of classical systems which are governed by stochastic differential equations of the type defined by (2.1). They are applicable to a much broader range of physical problems than the results of MSR. Although these equations also prove in general to be too complicated to solve directly, the advantages of such a description are obvious. First, these exact equations of motion exhibit all of the symmetries and conservation laws of the exact solutions. Second, these equations serve as a starting point for several different systematic perturiution schemes.

The functional integral, approach provides a natural and direct 
derivation of the closed schwinger and Dyson equations for deterministic interactions and linear random forces. If we assume arbitrary random forces (2.46) gives in general

$$
\begin{aligned}
H=\hat{\psi}(1)\left[\bar{U}_{1}(1)\right. & +\bar{U}_{2}(12) \psi(2)+\bar{U}_{3}(123) \psi(2) \psi(3)+\ldots \\
& \left.+\bar{U}_{n}(1 \ldots n) \psi(2) \ldots \psi(n)+\delta\left(t_{1}-t_{0}\right) \bar{\psi}_{0}(1)\right] \\
& +\ln \left(\exp \hat{\psi}(1) \tilde{U}_{1}(1)\right\rangle \\
= & H_{0}+C\{\hat{\psi}\}
\end{aligned}
$$

$\mathrm{H}_{0}$ represents the deterministic forces, interactions, and initial conditions and $\mathrm{C}$ is the cumulant functional

$$
c\{\psi\} \equiv \sum_{n=1}^{\infty} \frac{1}{n !}[\hat{\psi}(1) \ldots \hat{\psi}(n)]\langle\langle(\mathcal{O}(1) \ldots \widetilde{U}(n))\rangle
$$

where $\langle(\ldots)\rangle$ is the cumulant average of the random force. (26) For Gaussian random forces $c\{\hat{\psi}\}=\hat{\psi}(1)\left\langle\langle\widetilde{U}(1)\rangle+\frac{1}{2} \hat{\psi}(1) \hat{\psi}(2)\langle\langle\widetilde{U}(1) \tilde{U}(2)\rangle\rangle\right.$. When $(2.49)$ is inserted into Equations $(2.44),(2.45),(2.47)$, and (2.49), we can easily recover the Schwinger and Dyson equations derived by MSR which are written in matrix form in (2.19) and (2.20). In the following sections the statistical dynamical equations (2.44)-(2.48) will be explicitly determined for a variety of important physical problems. 
3. APPLICATIONS OF THE FUNCTIONAL INTEGPAL FORMALISM

A. Nor-Gaussian initial corditions.

Deker ${ }^{(6)}$ has recently observed that the MSR procedure cannot describe the evolution of systems with non-Gaussian initial conditions. He proposed a modification of the MSR formali in and suc-ceeded in deriving the "spurious" interactions which are generated by the cumulants of the random initial conditions.

Deker's results are easily recovered as a special case of our functional integral description of general stochastic differential equations. Without loss of generality, consider a classical system described by a differential equation of the form of (2.1) with deterministic forces and interactions but random initial conditions. The random initial condition is treated like an instantaneous linear random force. Using Equation $(2,46)$ for the averaged Hamiltonian we can write down the answer immediately

$$
\begin{aligned}
H=\hat{\psi}(1)\left[\overline{\mathrm{U}}_{1}(1)\right. & \left.+\ldots+\overline{\mathrm{U}}_{\mathrm{n}}(1 \ldots n) \psi(2) \ldots \psi(n)+\delta\left(t_{1}-t_{0}\right) \bar{\psi}_{0}(\underline{\sim})\right] \\
& +\ln \left\langle\exp \hat{\psi}(1) \widetilde{\psi}_{0}(1) \partial\left(t_{1}-t_{0}\right)\right\rangle \\
= & H_{0}+\sum_{n=1}^{\infty} \frac{1}{n !} \hat{\psi}(1) \delta\left(t_{1}-t_{0}\right) \ldots \hat{\psi}(n) \delta\left(t_{n}-t_{0}\right\rangle\left\langle\left\langle\widetilde{\psi}_{0}(1) \ldots \tilde{\psi}_{0}(\mathrm{n})\right\rangle\right\rangle
\end{aligned}
$$

where we have expanded out the cumulant function in terms of the cumulant averages of the random initial conditions. The corrections to $\mathrm{H}_{0}$ are the "spurious" interactions which were derived by Deker. (6) When $H$ is inserted into $(2.44),(2.45),(2.47)$, and (2.48) we get a complete description for the statistical dynamics of systems with arbitrary initial conditions. 
B. Multiplicative Random Forces .

Now consider a classical system with stochasic interactions $\hat{U}_{n}(1 \ldots n)$. For the purposes of illustration we will examine the case with $\widetilde{U}_{2}(12) \neq 0$. The results are easily generalized to systems with many stochastic interactions and using the results of Section 3.A to systems with random initial conditions as well.

Deker and Haake ${ }^{(3)}$ were the first to modify the MSR formalism to deal with problems of this kind. They treat the random force in the interaction $\tilde{U}_{2}(12)$ as a separate field on an equal footing with $\psi$ and $\hat{\psi}$.

A different approach to this problem has also been developed by Phythian (14) using an elegant method based on the Novikov theorem, (27) Phythian's method avoids the mixed averages of $\psi, \hat{\psi}$, and $\widetilde{\mathrm{U}}_{2}$ which result in Deker and Haake's approach since the Novikov theorem decouples the statistics of $\hat{U}_{2}^{\prime}$ from the statistics of $\psi$. Unfortunately, the Novikov theorem is restricted to Gaussian random forces and both methods require that the interactions be local.

The functional integral method provides a generalization of the Novikov theorem to nonlccal random interactions with arbitrary statistics. Furthermore, sınce the results of Deker and Haake are also easily recovered by treating the random force as an additional field, our method serves to unify these disparate approaches.

Once again (2.46) enables us to write down the answer

$$
\begin{aligned}
H & =\hat{\psi}(1)\left[\bar{U}(1)+\ldots+\bar{U}_{n}(1 \ldots n) \psi(2) \ldots \psi(n)\right]+\ln \left\langle\exp \hat{\psi}(1) \widetilde{U}_{2}(12) \psi(2)\right\rangle \\
& =H_{0}+C(\hat{\psi}(1) \psi(2)\}
\end{aligned}
$$


where the cumulant functional ${ }^{(26)}$ is given by

$$
c\{\hat{\psi}(1) \psi(\overline{1})\} \equiv \sum_{n=1}^{\infty} \frac{1}{n !} \hat{\psi}(1) \psi(\overline{1}) \ldots \hat{\psi}(n) \psi(\bar{n}) \gamma_{2}^{n}(1 \bar{I} \ldots n \bar{n})
$$

and

$$
\gamma_{2}^{n}(1 \bar{I} \ldots n \vec{n}) \equiv\left\langle\left(\tilde{U}_{2}(1 \overline{1}) \ldots \tilde{\mathrm{U}}_{2}(n \bar{n})\right\rangle\right)
$$

When (3.2) is inserted into bquations $(2.44),(2.45),(2.47)$, and (2.48) we arrive at a complete statistical description of the dynamics of systems with multiplicative random forces with arbitrary statistics.

Note that the random focce $\widetilde{U}_{2}$ appears explicitly only inside the cumulant averages $(3,4)$. Consequently, our approach decouples

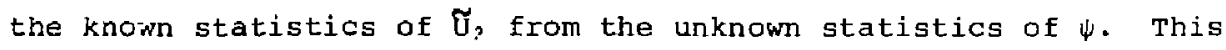
separation of the statistics has important practical advantages.

Similar results for instantaneous (local) random interacticis have been derived by Deker ${ }^{(6)}$ using an operator approach and by Phythian (11) using the functional integral formalism. ilowever, Phythian us - a difiwrent discretization corresponding to $\alpha_{2}=1$ and $\beta_{2}=0$ in ( 2.28$)$. This gives more complicated equations of motion because the Jacobian is a functional of $\psi$. Since the different discretizations are equivalent, Phythian's equations reduce to our simpler results.

If the random interactions are Gaussian then the schwinger and Dyson equations simplify considerably. Without loss of 
generality we neglect the deterministic forces and interactions. Then the statistical equations of motion are:

$$
\begin{aligned}
& \langle\dot{\psi(I)}\rangle_{c}-\left\langle\left\langle\tilde{U}_{2}(I I I)\right\rangle\langle\psi(\bar{I})\rangle_{c}\right. \\
& -\left\langle\left\langle\left(\widetilde{U}_{2}(1 \bar{I}) \widetilde{U}_{2}(2 \overline{2})\right\rangle\right\rangle\left\langle\frac{\psi(\bar{I}) \hat{\psi}(2) \psi(\overline{2})}{2}\right\rangle\right. \\
& -\zeta(1)=\delta\left(t_{1}-t_{0}\right) \Psi_{0}(1) \\
& -\langle\dot{\hat{\psi}}(1)\rangle_{\mathrm{c}}-\left\langle\left\langle\tilde{U}_{2}(\bar{I} I)\right\rangle\langle\hat{\psi}(\bar{I})\rangle_{c}\right. \\
& -\left\langle\left\langle\tilde{O}_{2}(\overline{1} 1) \tilde{U}_{2}(2 \overline{2})\right\rangle\right\rangle \frac{\langle\hat{\psi}(\overline{1}) \hat{\psi}(2) \psi(\overline{2})\rangle}{2} \\
& -n(1)=0 \\
& \frac{\partial}{\partial t_{!}}\left\langle\psi(1) \psi\left(I^{\prime}\right)\right\rangle_{c}-\left\langle\left\langle\tilde{U}_{2}(1 \bar{I})\right\rangle\right\rangle\left\langle\psi(\bar{I}) \psi\left(I^{\prime}\right)\right\rangle_{c} \\
& -\left\langle\left(\tilde{U}_{2}(1 \bar{I}) \tilde{U}_{2}(\langle\overline{2})\rangle\right\rangle\left[\frac{\left\langle(\bar{I}) \hat{\psi}(2) \psi(\overline{2}) \psi\left(I^{\prime}\right)\right\rangle}{z}\right\rangle\right. \\
& \left\langle\because \frac{\overline{1}, \hat{\psi}\{2\rangle+\{\overline{2}\}\rangle\langle\psi\{1 \cdot\rangle\rangle\}}{\mathrm{z}^{2}}=0\right. \\
& \frac{\partial}{\partial t_{1}}\left\langle\psi(1) \hat{\psi}\left(I^{\prime}\right)\right\rangle_{c}-\left\langle\left\langle\widetilde{U}_{2}(I \bar{I})\right\rangle\left\langle(\bar{I}) \hat{\psi}\left(I^{\prime}\right)\right\rangle_{c}\right. \\
& -\left\langle\left\langle\widetilde{U}_{2}(1 \overline{1}) \tilde{U}_{2}(2 \overline{2})\right\rangle\right\rangle\left\langle\frac{\left\langle\psi(\bar{I}) \hat{\psi}(2) \psi(\overline{2}) \hat{\psi}\left(I^{\prime}\right)\right\rangle}{2}\right. \\
& \left.-\langle\psi(\bar{I}) \hat{\psi}(2) \psi(\overline{2})\rangle\left\langle\hat{\psi}\left\langle I^{\prime}\right)\right\rangle\right]=\delta(1-1 \cdot) .
\end{aligned}
$$

These equations can be formally closed by expanding the three- and four-point correlations in terms of the connected Green's 
functions and then writing the connected Green's functions in terms of the two-point connected Green's functions and the three-point and four-point vertex functions.

If we neglect the three- and four-point connected Green's functions then this "Gaussian" approximation gives

$$
\begin{aligned}
& \frac{\partial}{\partial t_{1}}\left\langle\psi(1) \psi\left(I^{\prime}\right)\right\rangle_{c}-\left\langle\langle \tilde { U } _ { 2 } ( 1 \overline { I } ) \rangle \left\langle\langle(\bar{I}) \psi(1 \cdot)\rangle_{c}\right.\right. \\
& -\left\langle\left(\tilde{U}_{2}(I \bar{I}) \widetilde{U}_{2}\langle 2 \overline{2})\right\rangle\right\rangle\langle\psi(\bar{I}) \hat{\psi}(2)\rangle_{c}\left\langle\psi(\bar{L}) \psi\left(I^{\prime}\right)\right\rangle_{c}=0 . \\
& \frac{\partial}{\partial t_{1}}\left\langle\psi(1) \hat{\psi}\left(I^{\prime}\right)\right\rangle_{c}-\left\langle\left\langle\tilde{U}_{z}(1 \bar{I}]\right\rangle\right\rangle\langle(\bar{I}) \hat{\psi}(1 \cdot)\rangle_{c} \\
& -\left\langle( \tilde { U } _ { 2 } ( 1 \overline { I } ) \tilde { U } _ { 2 } ( 2 \overline { 2 } ) \rangle \left\langle\langle\psi(\bar{I}) \hat{\psi}(2)\rangle_{C}\left\langle\psi(\overline{2}) \hat{\psi}\left(I^{\prime}\right)\right\rangle_{C}=\delta\left(1-1^{\prime}\right),(3.10)\right.\right.
\end{aligned}
$$

These renormalized equations of motion for the fluctuation and response functions are identical to those derived in the lirect Intexaction Approximation(13) (DIA) using the methods of Deker and Haake. Krommes, Kleva, and Oberman (13) have applied the approach of Deker and Haake to the problem of particle motion in a stochastic magnetic field which was outlined in Section 2.A.(ii). Unfortunate$1 y$, because of complications related to the appearance of mixed averages of $\psi$ and $\widetilde{\mathrm{U}}_{2}$, they were unable to complete the problem beyond the DIA. Although the equations of motion (3.5) and (3.9) of both approaches are identical in lowest order renormalized perturbation theory (DIA), our new results (3.7) and (3.8) arroid the complications of mixed averages to all orders in the perturbation theory. The problem of particle motion in stochastic magnetic 
fields will be pursued further using (3.7) and (3.8) in a subsequent paper.

C. Nonlocal Interactions.

one of the distinct advantages of the functional integral formalism is that there are no restrictions to local or instantaneous interactions. The results of section 2.C are valid for any nonlacal but causal interaction $U_{n}(1 \ldots n)$. Consequently, this theory extends the modern methods of renormalized perturbation theory to a large new class of problems.

Many equations of this class, which originate from second or higher order differential equations, can also be written as a system of first order differential equations witj local interactions by extending the number of fields. This system of differential equations can be formally solved using the MSR formalism. However, the complications of the adaitional fields are easily avoided by dealing directly with a single differential equation with nonlocal interactions.

Some examples of important problems for which formal solutions can be obtained using the functional integral formalism, are wave prcpagation in random media, and the nonlinear theory of electromagnetic plasma turbulence. The structure of the dynamical equations for both problems was outlined in Section 2.A. In this section we will study the theory of electromagnetic plasma oscillations and derive the electromagnetic dispersion tensor(28) in lowest order renormalized perturbation theory. A detailed discussion of the stochastic wave equation will be reserved for a future publication. 
Krommes and Kleva ${ }^{(12)}$ have succeeded in calculating the dielectric tensor for electrostatic oscillations in a turbulent plasma using the methods of MSR. However, they were unable to apply their theory directly to the electromagnetic problem because of the restriction of the MSR approach tc instantaneous interactions. Using the functional integral approach it is easy to extend krommes and Kleva's resuits to the electromagnetic case.

In Section 2.A. (iv) we showed that the vlasov-Maxwell equations can be written in the form of (2.1) with non local deterministic interactions. The statistical averages arise either from the assumption of random initial conditions or random phases. The corresponding stochastic differential equation is

$$
\frac{\partial \psi(1)}{\partial t_{1}}-\bar{U}_{2}(12) \psi(2)-\bar{U}_{3}(123) \psi(2) \psi(3)=\delta\left(t_{1}-t_{0}\right) \psi_{0}(1) \text {. }
$$

The effective Hamiltonian is given by $(2.46)$

$$
H= \begin{cases}H_{0}, & \text { random phases } \\ H_{0}+\widetilde{\mathrm{H}}, & \text { random initial conditions }\end{cases}
$$

where $H_{0}=\hat{\psi}(1)\left[\bar{U}_{2}(12) \psi(2)+\bar{U}_{3}(123) \psi(2) \psi(3)+\delta\left(t_{1}-t_{0}\right) \bar{\psi}_{0}(1)\right]$ and $\widetilde{H}=\ln \left\langle\exp \tilde{\psi}(1) \delta\left(t_{1}-t_{0}\right) \widetilde{\psi}_{0}(\underline{\sim})\right\rangle$.

Substituting $\mathrm{H}$ into $(2.44),(2.45),(2.47)$, and $(2.4 \mathrm{~B})$ we get the statistical equations of motion for the mean field, the fluctuation function and the response function: 


$$
\begin{aligned}
\frac{\partial}{\partial t_{1}}\left\langle\frac{\psi(1)}{\mathrm{z}}-\overline{\mathrm{U}}_{2}(12)\right. & \frac{\langle\psi(2)\rangle}{\mathrm{z}}-\overline{\mathrm{U}}_{3}(123) \frac{\langle\psi(2) \psi(3)\rangle}{\mathrm{z}} \\
& -\zeta(1)=\left\{\begin{array}{l}
\delta\left(\mathrm{t}_{1}-\mathrm{t}_{0}\right) \bar{\psi}_{0}(1), \text { random phases } \\
\frac{1}{\mathrm{z}}\left\langle\frac{\delta}{\delta \hat{\psi}(1)} \tilde{\mathrm{H}}\right), \begin{array}{c}
\text { random initial } \\
\text { conditions }
\end{array}
\end{array}\right.
\end{aligned}
$$

$$
\begin{aligned}
& \frac{\partial}{\partial t_{1}}\left\langle\psi(1) \psi\left(1^{\prime}\right)\right\rangle_{\mathrm{c}}-\overline{\mathrm{U}}_{2}(12)\left\langle\psi(2) \psi\left(1^{\prime}\right)\right\rangle_{\mathrm{c}} \\
& -\overline{\mathrm{U}}_{3}(123)\left[\frac{\left\langle\psi(2) \psi(3) \psi\left(1^{\prime}\right)\right\rangle}{\mathrm{z}}-\frac{\left.\left\langle\psi\left(1^{\prime}\right)\right\rangle\langle\psi(2) \psi(3)\rangle\right]}{\mathrm{z}^{2}}\right. \\
& \left\{\begin{array}{l}
0, \text { random phases } \\
\frac{1}{z}\left\langle\psi\left(1^{\prime}\right) \frac{\delta}{\delta \hat{\psi}(1)} \widetilde{\mathrm{H}}\right\rangle-\left(\frac{\psi\left(1^{\prime}\right)}{\mathrm{z}}\right) \frac{1}{\mathrm{z}}\left(\frac{\delta}{\delta \hat{\psi}(1)} \tilde{\mathrm{H}}\right)
\end{array}\right. \\
& \text { random initial conditions. }
\end{aligned}
$$

$$
\begin{aligned}
& \frac{\partial}{\partial t}\left\langle\psi(1) \hat{\psi}\left(1^{\prime}\right)\right\rangle_{c}-\bar{U}_{2}(12)\left\langle\psi(2\rangle \hat{\psi}\left(I^{\prime}\right)\right\rangle_{c} \\
& \quad-\bar{U}_{3}(123)\left[\frac{\left\langle\psi(2) \psi(3) \hat{\psi}\left(1^{\prime}\right)\right\rangle}{z}-\frac{\left.\left\langle\hat{\psi}\left(I^{\prime}\right)\right\rangle\langle\psi(2) \psi(3)\rangle\right]}{z^{2}}\right. \\
& \quad=\delta\left(1-I^{\prime}\right)
\end{aligned}
$$


For Gaussian random initial conditions the right hand sides of (3.15) and (3.16) simplify further. For $\eta, \zeta \rightarrow 0$

$$
\begin{aligned}
& \frac{1}{z}\left\langle\frac{\delta}{\delta \hat{\psi}(1)} \tilde{\mathrm{H}}\right\rangle \equiv \frac{\left\langle\widetilde{\psi}_{0}(\underset{\tau}{\sim})\right\rangle \delta\left(t_{1}-t_{0}\right)}{\mathrm{z}} \\
& \frac{1}{\mathrm{z}}\left\langle\psi\left(1^{\prime}\right) \frac{\delta}{\delta \hat{\psi}(1)} \widetilde{\mathrm{H}}\right\rangle-\left\langle\frac{\left\langle\left(1^{\prime}\right)\right\rangle}{\mathrm{z}^{2}}\left\langle\frac{\delta}{\delta \hat{\psi}(1)} \widetilde{\mathrm{H}}\right\rangle\right. \\
& =\left\langle\psi\left(1^{\prime}\right) \tilde{\psi}(2)\right\rangle\left\langle\left\langle\dot{\psi}_{0}(2) \tilde{\psi}_{0}(1)\right\rangle\right\rangle \\
& \times \delta\left(t_{2}-t_{0}\right) \delta\left(t_{1}-t_{0}\right)
\end{aligned}
$$

where we have used $\langle\hat{\psi}(1)\rangle \mid \equiv 0$ and $z \mid \equiv 1$.

$$
\eta, \zeta=0 \quad \eta, \zeta=0
$$

In order to close $(3.13),(3.14),(3.15)$, and $(3: 16)$ we first

express the three-point correlation functions in terms of the connected Green's functions. The resulting equatiors for the fluctuation function $C(12) \equiv\langle\psi(1) \psi(2)\rangle_{\mathrm{C}}$ and response function $\mathrm{R}(12) \equiv\left\langle\psi(1) \hat{\psi(2)\rangle_{\mathrm{C}}}\right.$ as $\eta, \zeta \rightarrow 0$ are

$$
\begin{aligned}
& \frac{\partial}{\partial t_{1}} c\left(11^{\prime}\right)-\bar{U}_{2}(12) c\left(2 I^{\prime}\right)-\bar{U}_{3}(123)\langle\psi(2)\rangle c\left(3 I^{\prime}\right) \\
& -\overline{\mathrm{U}}_{3}(123)\langle\psi(3)\rangle c\left(21^{\prime}\right) \\
& -\overline{\mathrm{U}}_{\mathrm{3}}(123)\left\langle\psi(2) \psi(3) \psi\left(1^{\prime}\right)\right\rangle_{\mathrm{c}} \\
& =\left\{\begin{array}{l}
0, \text { random phases } \\
R(I ' 2) c\left(\underset{\sim}{2}, t_{0} i \underset{\sim}{1}, t_{0}\right) \delta\left(t_{2}-t_{0}\right) \delta\left(t_{1}-t_{0}\right), \\
\quad \text { random initial conditions. }
\end{array}\right.
\end{aligned}
$$




$$
\begin{aligned}
\frac{\partial}{\partial t_{1}} R\left(1 I^{\prime}\right) & -\bar{U}_{2}(12) R\left(2 I^{\prime}\right)-\bar{U}_{3}(123)\langle\psi(2)\rangle R\left(31^{\prime}\right) \\
& \left.-\bar{U}_{3}(123)\langle\psi(3)\rangle\right)^{\prime}\left(21^{\prime}\right) \\
& -\bar{U}_{3}(123)\left\langle\psi(2) \psi(3) \hat{\psi}\left(1^{\prime}\right)\right\rangle_{\mathrm{C}} \\
& =\delta\left(1-1^{\prime}\right) .
\end{aligned}
$$

The three-point connected Green's functions are in turn expressed in terms of the two-point connected Green's functions and the three-point vertex funciion $\Gamma_{3}$. Let $\Phi_{i}=\left(\begin{array}{l}\Phi_{1} \\ \Phi_{2}\end{array}\right)=\left(\begin{array}{l}\psi \\ \psi\end{array}\right)$, then using furctional chain rule, Equation (2.22) gives

$$
\begin{aligned}
\Gamma_{j}^{1 \pi n}(\overline{2} \overline{3} \bar{l}) \equiv & {\left[\left\langle\Phi_{i}(2) \Phi_{I}(\overline{2})\right\rangle_{c}\left\langle\phi_{j}(3) \phi_{m}(\overline{3})\right\rangle_{c}\left\langle\phi_{k}(I ') \phi_{n}(\bar{I})\right\rangle_{c}\right]^{-1} } \\
& \times\left\langle\Phi_{i}(2) \Phi_{j}(3) \phi_{k}(1 ')\right\rangle_{c} .
\end{aligned}
$$

since on $y_{y} \Gamma_{3}^{212}(\overline{2} \overline{3} \bar{l}), \Gamma_{3}^{121}(\overline{2} \overline{3} \bar{l})$, and $\Gamma_{3}^{112}(\overline{2} \overline{3} \bar{l})$ are nonvanishing, we are left with

$$
\begin{aligned}
& \left\langle\psi(2) \psi(3) \psi\left(1^{\prime}\right)\right\rangle_{C} \equiv R(2 \overline{2}) C(3 \overline{3}) c(1, \overline{1}\rangle \Gamma_{3}^{2} I^{1}(\overline{2} \overline{3} \overline{1}) \\
& +C(2 \overline{2}) R(3 \overline{3}) C(1 \cdot \overline{1}) \Gamma_{3}^{2} 1(\overline{2} \overline{3} \overline{1}) \\
& +C(2 \overline{2}) C(3 \overline{3}) R\left(L^{\prime} \bar{L}\right) \Gamma_{3}^{1}{ }^{12}(\overline{2} \overline{3} \overline{1}), \\
& \left\langle\psi(2) \psi(3) \hat{\psi}\left(1^{*}\right)\right\rangle_{C} \equiv R(2 \overline{2}) C(3 \overline{3}) R\left(\overline{1} I^{\prime}\right) \Gamma_{3}^{2} 1 /(\overline{2} \overline{3} \overline{1}) \\
& +C(2 \overline{2}) R(3 \overline{3}) R\left(\bar{I} 1 \cdot 1 \Gamma_{3}^{12}\{\overline{2} \overline{3} \bar{I}\} .\right.
\end{aligned}
$$


In lowest order renormalized perturbation theory (DIA) (2.22) and (3.19) give

$$
\begin{aligned}
& \Gamma_{3}^{211}(\overline{2} \overline{3} \bar{I})=\bar{U}_{3}(\overline{2} \overline{3} \bar{I})+\overline{\mathrm{U}}_{3}(\overline{2} \bar{I} \overline{3}), \\
& \Gamma_{3}^{121}(\overline{2} \overline{3} \overline{1})=\overline{\mathrm{U}}_{3}(\overline{3} \overline{2} \overline{1})+\overline{\mathrm{U}}_{3}(\overline{3} \overline{\mathrm{I}} \overline{2}), \\
& \Gamma_{3}^{112}(\overline{2} \overline{3} \overline{1}) \simeq \overline{\mathrm{U}}_{3}(\overline{\mathrm{I}} \overline{3} \overline{2})+\overline{\mathrm{U}}_{3}(\overline{\mathrm{I}} \overline{\mathrm{z}} \overline{3}) .
\end{aligned}
$$

Following Krommes and Kleva(12) we then identify

$$
\begin{aligned}
& \Sigma(1 \bar{I})=\overline{\mathrm{U}}_{3}(123) \mathrm{R}(2 \overline{2}) C(3 \overline{3})\left[\overline{\mathrm{U}}_{3}(\overline{2} \overline{3} \overline{\mathrm{I}})+\overline{\mathrm{U}}_{3}(\overline{2} \overline{\mathrm{I}} \overline{3})\right] \\
& +\overline{\mathrm{U}}_{3}(122) \mathrm{R}(3 \overline{3}) \mathrm{C}(2 \overline{2})\left[\overline{\mathrm{U}}_{3}(\overline{3} \overline{\mathrm{L}} \overline{\mathrm{I}})+\overline{\mathrm{U}}_{3}(\overline{3} \overline{\mathrm{I}} \overline{2})\right] \text {, } \\
& \widetilde{\Sigma}(1 \bar{I})=\bar{U}_{3}(12 \Xi) C(2 \overline{2}) C(3 \overline{3})\left[\overline{\mathrm{U}}{ }_{3}(\bar{I} \overline{3} \overline{2})+\overline{\mathrm{U}}_{3}(\bar{I} \overline{2} \overline{3})\right], \\
& {\left[g_{0}\right]^{-1}(12)=\delta(1-2) \frac{\partial}{\partial t_{2}}-\bar{U}_{2}(12)-\bar{U}_{3}(132)\langle\psi(3)\rangle}
\end{aligned}
$$

Finally, using $(3.21)-(3.28)$, the dynamical equations can be written compactly as

$$
\begin{aligned}
{\left[g_{0}\right]^{-1}(12\rangle C\left(2 I^{\prime}\right) } & -\bar{U}_{3}(123)\langle\psi(3)\rangle C\left(2 I^{\prime}\right) \\
& -\Sigma(1 \bar{I}) C\left(\overline{1} I^{\prime}\right)-\tilde{\Sigma}(1 \bar{I}) R\left(I^{\prime} \bar{I}\right) \\
& =\left\{\begin{array}{l}
0, \text { random phases } \\
C\left(1 I^{\prime}\right) \delta\left(t_{1}-t_{0}\right), \text { random initial conditions }
\end{array}\right.
\end{aligned}
$$

$$
\begin{aligned}
{\left[g_{0}\right]^{-1}(12) R\left(21^{\prime}\right) } & -\bar{U}_{3}\left(12^{*} ;(\psi(3)) R\left(21^{\prime}\right)\right. \\
& -\Sigma(1 \overline{1}) C(\bar{I} 1)=\delta\left(1-1^{\prime}\right)
\end{aligned}
$$


Krommes and Kleva showec that in the electrostatic problem $\mathrm{R}$ can be considered as the renormalized propagator for a shielded test particle where the shielding is determined explicitly by a non linear, renormalized dielectric function. Analogous results can be shown for the electromagnetic case with the dielectric function replaced by the dispersion tensor.

Using the explicit representation of $\overrightarrow{\mathrm{u}}_{3}$ given by $(2.16)$, we can write $\sum$ as the sum of two types of terms

$$
\begin{aligned}
& \Sigma(1 \bar{I})=-\left\{\left[\frac{\partial}{\partial V_{1}} R(12)\right] \cdot \underline{L}(13) \_(2 \overline{3}) C(3 \overline{3})\right. \\
& \left.+\frac{L}{\sim}(13) \mathrm{R}(32)\left[\frac{\partial}{\partial \mathrm{\sim}_{2}} \cdot L(2 \overline{3}) \mathrm{C}(1 \overline{3})\right]\right\} \cdot \frac{\partial}{\partial \mathrm{V}_{2}} \delta(2-\overline{\mathrm{I}}) \\
& -\int\left[\frac{\partial}{\partial \underset{\sim}{v_{1}}} R(12)\right] \cdot \underset{\sim}{L}(13) \frac{\partial}{\partial{\underset{\sim}{2}}^{2}} C(32)
\end{aligned}
$$

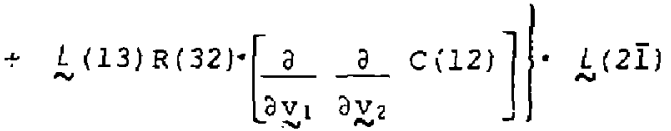

$$
\begin{aligned}
& \equiv \Sigma^{\prime}(1 \overline{1})+\left[\frac{\partial}{\partial \mathbf{v}_{2}} \delta \psi(12)\right] \cdot \underset{\sim}{\sim}(2 \bar{I}) .
\end{aligned}
$$

Using a more compact notation, Equation (3.31) for the averaged response function takes the form

$$
\mathrm{g}^{-1} \mathrm{R}+\frac{\partial}{\partial \mathrm{v}}[\langle\psi\rangle+\delta \psi] \cdot \underset{\sim}{L} \mathrm{R}=1 .
$$

One piece of the nonlinear interaction term $\Sigma$ renormalizes the bare propagator 9 , 


$$
g^{-1}(12) \equiv\left[g_{0}\right]^{-1}(12)+\Sigma^{\prime}(12)
$$

and the other piece modifies the mean background distribution

$$
\bar{\psi}(12) \equiv\langle\mu(1)\rangle \delta(1-2\rangle+\delta \psi(12) .
$$

Solving formally for $R$ we get

$$
L^{R}=\Delta^{-1}: L^{G}
$$

and

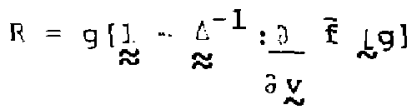

where the shielding is described by

$$
\hat{\approx}^{(21)}=\frac{1}{\approx}(2-1)+\hbar\left(2 \bar{z} \lg (\overline{2} \bar{l}) \frac{\partial}{\partial v^{i}} \bar{T}(\bar{l} 1) .\right.
$$

The electromagnetic dispersion tensor relates the average electromagnetic fields inside a stochastic dispersive medium, which result as a resporse to externally applied fields, to the perturbinu fields. Using the arguments of krommes and kleva ${ }^{\{12\}}$ it can be shown that $\Delta$ is a correct representation of the elo tromagnetic alspersios tensor.

The author has also considered this problem by exteriding the number of components of the classical field to make the interactions local. This allows the methods of MSR to be used. The results of this rather tedious calculation are identical to Equation (3.37).

Finall $y$, the reduction to linear theory can be shown, if we 
transform the disfersion tensor into a more familiar form which relates the total electric field to an applied external current. (28) In an isotropic mediun the longitudinal and transverse parts of the dispersion tensor decouple. In Fourier transform space

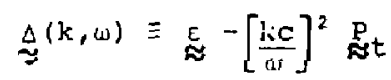

where

$$
\underset{\mathrm{Z}}{\mathrm{Z}} \equiv \delta_{i j}-\frac{k_{i} k_{j}}{k^{2}} \quad, i=1,2,3
$$

and

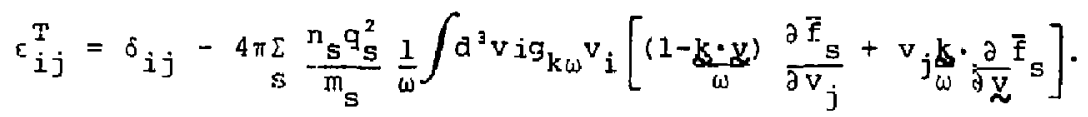

$g_{k,}$ is the kernel of the bare propagator (3.33) in Fourier space and $\overline{\mathrm{f}}$ is defined by (3.34). Equation (3.38) reduces to the linear dispersion tensor ${ }^{(2 g)}$ when the nonlinear terms $\Sigma^{\prime}$ in $g$ and $\delta f$ in $\overline{\mathrm{f}}$ are neglected. 
4. CONCLUSION

We have developed a functional integral formalism for the description of the statistical dynamics of a broad class of stachastic differential equations. The functional integral approach provides a natural and elegant derivation of all previous results based on the MSR operator formalism and extends these methods to classical systems with non..ocal interactions. Moreover, we emphasize that the functional integral results decouple the known statistics of the rangom forces, interactions, and initial conditions from the unknown statistics of the classical random fields.

Oux formal results are illustrated by an application in the theory of electromagnetic plasma turbulence. Using the functional integra] formalism for nonlocal interactions we have extended Krommes and Kleva's derivation of the nonlinear dielectric function for electrostatic plasina turbulence to the electromagnetic case. The resuling noninear dispersion tensor provides a formal basis for further work on the ronIinear evolution of plasma instabilities.

\section{ACKNOWLEDGMENTS}

The author is grateful to $C$. Oberman and $J$. Krommes for stimulating discussions.

This work was supported by the United States Department of Energy DE-ACO2-76-CHO3073. 


\section{REFERENCES}

1. P.C. Martin, E.D. Siggia, and H.A. Rose, Phys. Rev. A 8:423(1973).

2. J. Schwinger, Proc. Natz. Acad. Sci. 37:452(1951).

3. U. Deker and F. Haake, Phys. Rev. A $11: 2043$ (1975).

4. R. Phythian, J. Phys. A 9 :269(1976).

5. H.A. Rose, J. Stat, Phys. 20:415(1979).

6. U. Deker, Phys. Rev. A $19: 846(1979)$.

7. R.P. Feyman and A.R. Hibbs, Quantum Mechanios and Pati Integrals, (McGraw-Hill, New York, 1965)

8. R. Graham, in springer tracts in Modern physias, vol. 66 (Springer, New York, 1973).

9. C. DeDominicis, J. de Phys. Cl1:247(1976).

10. H.K. Janssen, 2. Phys. B2 $23: 377$.

11. R. Phythian, J. Phys. A10:777(2977).

12. J.A. "ommes and R.G. Kleva, Phys. Fl, 22: 2168(1979).

13. J.A. Krommes, R.G. Kleva, and C. Oberman, Princeton Flasra Physiaz Laboratirg Report PPPL-1389 (1978) (to be published).

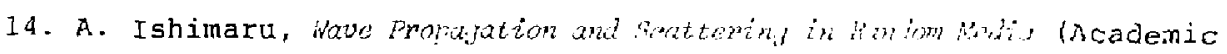
Press, New York, 1978).

15. U. Frisch, in Probabizistic Methods in Appind Afathometios, (Academic Press, New York, 1968).

16. J.D. Jackson, Classical Elecipolunamics, (Wiley, New York, 1975).

17. R+H. Kraichnan, J. Kath. Plizs. $2: 124(1961)$.

18. H.A. Rose, thesis (karvard, 1974) (unpublished).

19. D.J. Amit, Field Theory, Renomalization Group, and Criticat Phenomena, (McGraw-Hill, New York, 1978).

20. K.D. Friedrichs et. al., Integration of Functionals, courant I: stitute of Mathematical Sciences, New York, 1976). 
21. F. Langouche, D. Roekaerts, and F. Tirapegui, Physica 95A:252(1979). 22. E.S. Abers and B.W. Lee, Phys. Rep. 9:1(1973).

23. B. Jouvet and R. Phythian, Phys. Rev. Al9:1350(1.979).

24. A. Katz, Classical Mechanies, Quantum Mechanios, Field Theory, (Academic Press, New York, I965).

25. L. Garrido, D. Lurie, M. San Miguel, J. Stat. Phys. 21:313(1979).

26. R. Kubo, t. Phys. Soc. Jap. 17:1100(1962).

27. E.A. Novikov, Sav. Phys. J.E.T.P. 20:1299(1965).

28. S. Ichjmaru, Basic Principles of Plasma Physies: A Statistical Approach, (Benjamin, New York, 1973). 


\section{ALL CA TEGORIES}

12. Astew, Auburn Un versity, Alabaina

\$. T. Wu, Univ of Alabaina

Geophysical Institute, Univ. of Mlaska

G.L. Johnston, Sonoma St ate Univ, California

H. H. Kuetil, Ini v. of 5. California

Institute for Energy 5 tudies, Stanford University

4. D. rampbell, University of Florida

N. L. Oleson, University of South Florida

W. M. Stace $y$, Georgia Insti tute of Technology

Benjamin Ma, lowa State University

Magne Kristiansen, Texas Tech. University

W. L. Wiese, Nat'l Bureau of Standar ds, Wash., D.C.

Australian National University, Canberra

C.N. Wate on-Munro, Univ, of Sydney, Australia

F. Cap, Inst. for Theo. Physics, Austria

Dr.w. Heirder, Institute for Theoretical Ffysics

Technical University of Graz

Ecole Royale Milit aire, Bruxelies, Belgitum

D. Pa:umbo, C. European Comm, B-1049-Brussels

P.H. Sakanaka, instituto de Fisica, Campinas, Brazil

M.P. Bachynski, MPB Tech., Ste. Anne de Bellevue,

Quebec, Canada

C.R. James, University of Mberta, Canada

T.W. Johnston, INRS-Energic, Vareenes, Quebec

H. M. Skarsgard, Uni y. of Saskatchewar, Canada

Inst. of Physics, Academia Sinica, Peking,

People's R epublic of China

Inst. of Plasma Physics, t lef ei,

A ninwei Province, People's Republic of China

Library, Tsing Hua Univ., Peking, Pcople's

Republit: of Chi nil

ZhengwuLi. Sout hwestern inst of Phys., Leshan, Sichuan Province, Pcople's Republıc of China

Librarian, Cul ham Laboratory, Abingdon, England

M.M. Dupas Library, C.E.N.G, Grenoble, France

Central Res. Inst. for Physics, Hungary

S. R. Sharma, Iniv of Rajasthem, JAIPUR-4, india

R. Shi ngit, Mecrut : :ollege, Indi a

A.K. Sundaralln, Pa!s. Res. Lab., India

Bîblotecu, Frascu:l, Italy

Bibliotecal, Wilano, ltaly

G. Rostagni, I riv. Di Paduva, Padova, Italy

Preprint Library, Inst. de Fisica, Pisa, Italy

Library, Plasina Physics Lab., Gokas/o, Ujj, Japan

S. Mori, Japan A tonic Energy Res. Inst., Tokai-Mura

Rescar ch Information Center, Nagoya Univ., Japan

5. Shioda, Toleyo Insז. of Tech., Japan

Intst. of Space \& Aero. Sri., IJ niv, of Tokyo

T. Uchida, Univ, of Tokyo, Japan

H. Yanato, Tos hiba R. \& D, Center, Japan

M. Yoshikawa, JAEIRI, Tokai Res. Est. Japan

Dr. Tsuneo Nakakj ta, Tostiba Corporation,

Kawasaki-KuKawasaki, 210 Japan

N. Yajima, Kyushu Univ., Tapan

R. England, Univ. Nacional Auto nom a de Mexico

B. 5. Liley, Univ. of Waikato, New Zealand

5. A. Moss, Suab Univas Norge, Norway

J.A.C. Cabral, Univ. de Lisboa, Portugal

O. Petrus, AL.I. CUZA Univ "llomania

J. de Villiers, Atomic Energy Bd., South Africa

A. Malsrech, Comisaria De La Energy y Recoursos Minerales, Spain

Librar s, Royal Institute of Technology, Sweden

Cen. de Res. En Phys,Des Plasmas, Switzerland

Librarian, Fon-institust Voor Plasma-Fysica,

The Netherlands
Bibliothek, Sinttgart, West smany

R.D. Buhler, Univ, of Stuttgart, West Germany

Max-Planck-Inst, f ur PJasmaphysik, W. Germany

Nucl. Res. Estab., Jul ich, West Germany

K. Schindler, Inst. Fur Theo. Physik, W. Germany

\section{EXPERIMENTAL THEORETICAL}

M. H. Brennan, Flinders Univ, Austr "ia

H. Barnard, Univ. of British Col umbia, Canada

S. Screenivasan, U ni v, of Cal gary, Caviada

J. R adet, C.EN.-B.P., Fontena $y-a u x=R$ oses, France

Prof. Schatzman, Obser vatoire de Nice, France

5. C. Sharna, Univ of Cape Coast, Ghana

R. N. Aiyer, l-aser Section, Indra

B. Buti, Physical Res. Lab., India

L. K. Chavda, S. Gujarat Univ., India

I.M. Las Das, Banar as Hindu Uni v., India

S. Cuperman, Tel A viv Univ., Israel

E. Greenspan, Nuc. Res. Center, Israel

P. Rosenau, Israel Inst . of Tech., Israel

Int'l. Center for Theo. Physics, Trieste, Italy

I. Kawakarni, Nihon Univer sity, Japan

T. Nakayama, Ritsuneikan Univ., Japan

5. Nagao, Tohoku Univ., Japan

]. I. Sakai, Toyarna Univ., Japan

S. Tjotta, Univ. I Bergen, Norway

M.A. Hellberg, Univ, of Natal, South Africa

$H$. Wilhelms on, Chalmers Univ, of Tech., Sweden

Astro. Inst., Sonnenborgh Obs.,

The Netherlands

T. J. Boyd, Univ. College of North Wales

K. Hubner, Gniv. Meidelberg, wermany

H. J. Kaeppeler, Univ. of Stuttgart,

West Geritiany

K. H. Spatschek, Univ. Essen, west Germany

\section{EXPEIRINENTAL \\ ENGINEERING}

B. Grek, Unj v. du Quebec, Canada

P. Lukac, Komenskeho Univ., Czechoslovakia

G. Horikoshi, Nat'l Lab Jor High Energy Physics, Tsukuba-Gun, Japan

\section{EXPERIMENTAL}

F. J. Paoloni, Univ. of Woll sngong, Australia

J. Kistermaker, Forn lnst. fo: A tomic \& Molec. Physics, The Nitherlands

\section{THEORETIGAL}

F. Verheest, Inst + Vor Theo. Mech., Belgiun

J. Teichmann, Univ, of Montreal, Canada

T. Kahan, Univ. Paris Vll, France

R. K. Chhajlari, India

S. K. Trehan, Panjab Univ., India

T. Namikawa, Osaka City Uniw Japan

H. Nar tomi, Univ. of Hiroshima, Japan

Korea A toinic Energy Res. Inst , Korea

E. T. Karlson, Uppsala Uni v, Sweden

L. Stenflo, Univ. of UMEA, $\zeta$ weden

7. R. Sar af, New Uni $v_{*}$, Uni ted Kingdom 